\title{
Low-Complexity SCMA Detection for Unsupervised User Access
}

\author{
Christopher Husmann, Chathura Jayawardena, Amine Maaref, Pei Xiao \\ and Konstantinos Nikitopoulos
}

\begin{abstract}
Non-orthogonal multiple access schemes (NOMA), such as sparse code multiple access (SCMA), are among the most promising technologies to support massive numbers of connected devices. Still, to minimize the transmission delay and to maximize the utilization of the transmission channel, "grantfree" NOMA techniques are required that eliminate any prior information exchange between the users and the base-stations. However, if a large number of users transmit simultaneously in an "unsupervised" manner, (i.e., without any prior signaling for controlling the number of users and the corresponding transmission patterns), it is likely that a large number of users may share the same frequency-resource element, rendering the corresponding user detection impractical. In this context, we present a new multi-user detection approach, which aims to maximize the detection performance, with respect to given processing and latency limitations. We show that our approach enables practical detection for grant-free SCMA schemes that support hundreds of interfering users, with a complexity that is up to two orders of magnitude less than that of conventional detection approaches.
\end{abstract}

Index Terms-Non-Orthogonal Multiple Access (NOMA), Sparse Code Multiple Access (SCMA), Detection

\section{INTRODUCTION}

Code-domain NOMA schemes, such as sparse code multiple access (SCMA) [1], enable non-orthogonal access by allowing different users employing patterns (or codewords) that overlap (e.g., they share time-frequency resource elements). If the patterns overlap in a sparse manner in the time-frequency grid, as in SCMA, the efficient message passing algorithm (MPA) [2] can be used for joint multi-user detection. MPA utilizes a factor graph composed of variable nodes (VNs) and resource nodes (RNs), and performs multi-user detection by iteratively exchanging "soft" information between the RNs and the VNs [2]. Then, MPA's computational complexity is dominated by the complexity required to calculate the soft information at each $\mathrm{RN}$ [3]. that can increases exponentially with the number of users that share the specific time-frequency element associated with a particular RN [3]. Consequently, reducing MPA complexity in the context of SCMA systems has received significant research attention. The authors in [4], for example, propose to execute MPA in the logarithmic domain (log-MPA) to transform the majority of the required complexity intensive multiplications into summations. Codebooks, specifically designed to reduce detection complexity have been introduced in [5], that effectively reduce the number of possible combinations a RN has to check. The authors in [6] leverage partial marginalization. In particular, after a predefined number of detection iterations, the soft information of the most reliable users is no longer updated. In the same context, a combination of MPA with list sphere decoding

\footnotetext{
Corresponding Author:
} k.nikitopoulos@surrey.ac.uk.
[7], [8] has been proposed in [3]. While the aforementioned schemes achieve substantial complexity reductions compared to the original MPA, their complexity requirements are still impractical for a large number of users. Recently, massively parallel detection schemes have been proposed to cope with the increased complexity and latency requirements in the case of large number of users [9]. Still, such approaches do not exploit the inherent MPA properties of unsupervised access, for complexity reduction, that is the focus of this work.

Other major obstacles underpinning massive connectivity are related to the latency and signaling overhead of the channel access procedures [10]. Grant-free NOMA schemes aim to reduce this latency and signaling overhead, by realizing access techniques that do not require an information exchange between the transmitter and the receiver prior to the payload transmission. In such "unsupervised" access, we assume that the users transmit their payloads without a prior grant, or other kind of "user-supervision", procedure. However, and similarly to [11], [12], while the users are unsupervised in the way they access the medium, they can still be synchronized by means of periodically broadcasted reference signal transmissions [12]. In addition, similarly to [11], in such transmissions, the number of active users and their corresponding transmission channels can be estimated by employing traditional channel estimation procedures that utilize orthogonal (across the users) pilot sequences. In this context, SCMA-based approaches are particularly well suited for grant-free access schemes.However, in the absence of "user-supervision" there is no control on the maximum number of users that may share a time-frequency resource element. Thus, it is likely that a large number of interfering users share the same resource element with the complexity required for performing MPA detection being well beyond the processing capabilities of our dedicated hardware.

Here we present a simple but efficient MPA-based detection approach which aims to maximize the detection performance with respect to given processing and latency limitations despite the fact that large numbers of users may interfere at several time-frequency resource elements. While our proposed approach is generally applicable to any scenario where MPA is employed, we herein, focus on SCMA-based systems. We exploit two fundamental observations which, according to the best of our knowledge, are explored for the first-time in the context of MPA detection. First, we exploit the fact that while the processing of RNs related to time-frequency resources with few interfering users are less complex, the soft information provided by such RNs contributes, in average, more to the symbol decision (e.g., increase detection confidence) than the soft information provided by RNs with many interfering users. 
This is discussed in detail in Section II. The second observation is that, in an unsupervised access scheme, it is likely to have a large number of users interfering at a time-frequency resource element, but it is equally likely, to also have timefrequency resource elements where only a small number of users interfere. Therefore, low-complexity, and highly reliable soft information calculation can take place. Based on these observations our proposed approach prioritizes the processing of the RNs with small number of interfering users when the processing capabilities are not sufficient to employ traditional MPA. In addition, following the principles of [7], we also introduce a simplified metric to avoid unnecessarily spending processing on RNs that will not significantly contribute to the overall soft information reliability. Despite its simplicity, our approach can support hundreds of mutually interfering users with computational requirements that are orders of magnitude smaller than those of traditional MPA-based approaches.

\section{SCMA PRIMER}

In general, a SCMA transmitter spreads the user's $M$ information bits across a set of $K$ OFDM subcarriers. The available spreading patterns (i.e., codewords) they can be described by the $K$ dimensional vectors $\mathbf{x}$, with the components of $\mathbf{x}$ representing the complex signal transmitted on each subcarrier. Each of the $U$ transmitting users selects a codeword from a randomly chosen codebook. Then, all users synchronously transmit their codeword to the base station. Since, all users utilize the same $K$ OFDM subcarriers, the SCMA system is characterized by an overloading factor of $U / K$. An SCMA encoder maps the $M$ dimensional information bit vector $\mathbf{b}$ of a user to a codeword $\mathbf{x}$ belonging to the codebook $C$. This mapping function $C$ is defined as $f_{C}: \mathbb{B}^{M} \rightarrow X$ with $\mathbf{x}=f_{C}(\mathbf{b})$, where $X \in \mathbb{C}^{K}$ and has a cardinality of $2^{M}$. In principle, only $N$ (with $N<K$ ) components of the codeword $\mathbf{x}$ are non-zero, and each of them is selected from a complex symbol constellation (e.g. QAM-symbols). Codewords from different codebooks differ in, at least, one non-zero position, while all codewords of a specific codebook have common nonzero positions. Due to these properties, codewords of different codebooks overlap in a sparse manner, which enables efficient MPA-based multi-user detection [2]. The SCMA mapping function can be described as $f_{C}: \equiv \mathbf{V}_{C} \cdot g_{C}$ where the function $g_{C}$ maps the binary vector $\mathbf{b}$ to an $N$-dimensional complex constellation vector, and $\mathbf{V}_{C}$ performs the corresponding spreading across the OFDM subcarriers. In general, a SCMA system has in total $J=\left(\begin{array}{l}N \\ K\end{array}\right)$ distinct codebooks, each consisting of $2^{M}$ different $K$-dimensional codewords with $N$ non-zero components. As already described, we assume that each user $u$ selects randomly one codebook $C_{u}$ out of $J$ existing. By choosing the mapping function $f_{C_{u}}$ of the selected codebook $C_{u}$, the user $u$ maps its bit vector $\mathbf{b}_{u}$ onto the SCMA codeword $\mathbf{x}_{u}=f_{C_{u}}\left(\mathbf{b}_{u}\right)$. Then, the received signal $\mathbf{y}$ consisting of the mutually interfering signals of all $U$ users is given by

$$
\mathbf{y}=\sum_{u=1}^{U}\left(\operatorname{diag}\left(\mathbf{h}_{u}\right) \cdot \mathbf{x}_{u}\right)+\mathbf{z}
$$

where $\mathbf{h}_{u}$ is a $K$ dimensional vector with each element representing the frequency domain channel response of the $u^{\text {th }}$ user for a specific subcarrier, and $\mathbf{z}$ is an additive white Gaussian noise vector of $\mathcal{N}\left(0, \sigma^{2} \cdot \mathbf{I}\right)$. The interference pattern of SCMA systems can be represented by a factor graph. In particular, each of the $U$ transmitting users is represented by a variable node $(\mathrm{VN})$, and each of the $K$ occupied OFDM subcarrier is represented by one resource node $(\mathrm{RN})$. Unless specified otherwise, we denote the VN representing the $u^{t h}$ user as $V N_{u}$ and the $\mathrm{RN}$ representing the $k^{\text {th }}$ subcarrier as $R N_{k}$. During the decoding process, soft information related to the transmission probability of all possible codewords is iteratively exchanged between the connected VNs and RNs. In particular, a soft information message transmitted from $R N_{k}$ to $V N_{u}$ is denoted as $I_{R N_{k} \rightarrow V N_{u}}$ and a message from $V N_{u}$ to $R N_{k}$ is denoted as $I_{V N_{u} \rightarrow R N_{k}}$. All soft messages are initially set to zero. When $S$ users collide on the $k^{t h}$ subcarrier, the node $R N_{k}$ will be connected to $S$ different VNs. Then, the invertible function $f$ maps the indices of the VNs connected to $R N_{k}$ to integer numbers from 1 to $S$. The $S$ symbols that are transmitted on the $k^{\text {th }}$ subcarrier can, then, be described by the $S$-dimensional vector $\mathbf{x}_{R}$, where the $s^{t h}$ component of $\mathbf{x}_{R}$ represents the symbol transmitted from user $u=f(s)$ on the $k^{t h}$ subcarrier. Since each user selects one out of $2^{M}$ possible codewords, the set consisting of all possible $\mathbf{x}_{R}$ realizations, nominated as $\mathcal{X}_{R}$, has a cardinality of $2^{M \cdot S}$. Using the max-log approximation, the message related to the SCMA codeword component $\mathbf{x}_{u}(k)$ from $R N_{k}$ to $V N_{u}$, is now calculated as

$$
\begin{aligned}
& \min _{\substack{\mathbf{x}_{R} \in X_{R}, \mathbf{x}_{R}\left(f^{-1}\{u\}\right)=\mathbf{x}_{u}(k)}}\left\{d\left(\mathbf{x}_{R}\right)+\sum_{\substack{s=1, s \neq N^{-1}(u)}}^{S} I_{V N_{f(s)} \rightarrow R N_{k}}\left(x_{R}(s)\right)\right\} \\
& \left.\mathbf{x}_{u}(k)\right)=
\end{aligned}
$$

with

$$
d\left(\mathbf{x}_{R}\right)=\left\|\mathbf{y}(k)-\sum_{s=1}^{S} h_{f(s)}(k) \cdot \mathbf{x}_{R}(s)\right\|^{2}
$$

If $\mathcal{V}_{u}$ is the set of RNs connected to $V N_{u}$, then the soft messages from $V N_{u}$ to $R N_{k}$ regarding the codeword $\mathbf{x}_{u}$ is calculated as

$$
I_{V N_{u} \rightarrow R N_{k}}\left(\mathbf{x}_{u}(k)\right)=\sum_{l \in \mathcal{V}_{u}, l \neq k} I_{R N_{l} \rightarrow V N_{u}}\left(\mathbf{x}_{u}(l)\right),
$$

and the log-likelihood value of the SCMA codeword $\mathbf{x}_{u}$ is

$$
I_{u}\left(\mathbf{x}_{u}\right)=-\sum_{l \in \mathcal{V}_{u}} I_{R N_{l} \rightarrow V N_{u}}\left(\mathbf{x}_{u}(l)\right) .
$$

Then, the log-likelihood ratio (LLR) for the $m^{\text {th }}$ transmitted bit of user $u$ is calculated as

$$
\operatorname{LLR}\left(\mathbf{b}_{u}(m)\right)=\min _{\mathbf{x}_{-} \in \mathcal{B}_{m}^{-}}\left\{I_{u}\left(\mathbf{x}_{-}\right)\right\}-\min _{\mathbf{x}_{+} \in \mathcal{B}_{m}^{+}},\left\{I_{u}\left(\mathbf{x}_{+}\right)\right\},
$$

where the set $\mathcal{B}_{m}^{+}$consists of the codewords that are associated with $\mathbf{b}_{u}(m)=1$, and $B_{m}^{-}$is the set of codewords associated with $\mathbf{b}_{u}(m)=0$ [13]. To calculate $I_{R N_{k} \rightarrow V N_{u}}$, traditional MPA approaches evaluate (3) for all $2^{M \cdot S}$ elements in $X_{R}$. Since in grant-free access systems the number of colliding 


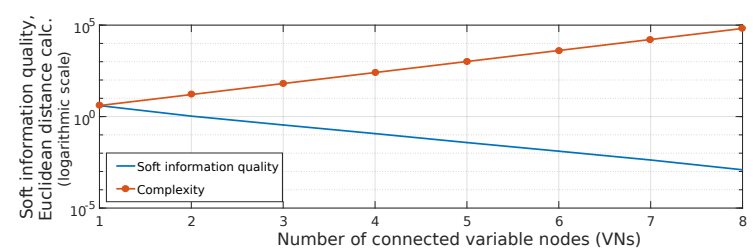

Fig. 1: Complexity (red) and "information quality metric" (blue) of a RN as a function of it's number connections to VNs. The cardinality of all codebooks is 4 .

users $S$ can significantly vary per resource element, the RNs with the largest number of interfering users will determine MPA's complexity requirements, which becomes impractical for large $S$ values. To overcome this problem, as discussed, our proposed approach is based on two fundamental observations that are visualized in Fig. 1. This figure shows that the required complexity to process a specific RN increases exponentially with the number of its connections to VNs or, equivalently, with the number of interfering users on the corresponding resource element. In addition, Fig. 1 shows that the average (absolute) LLR value difference between the most likely and the second most likely codewords in a codebook decreases when the number of interfering users on a resource element increases. Since the corresponding LLR values are related to the probability of each codeword to be the correct one [7], this difference is a good indicator of the receiver's decoding "confidence" in the specific codeword. Therefore, Fig. 1 reveals the fact that while the processing requirements for calculating the soft information of RNs related to large number of interfering users increases exponentially, still the corresponding contribution to the overall decoding confidence decreases.

\section{Proposed SCMA Detector}

Our proposed algorithm consists of two operations that work synergistically. First, our proposed $R N$ assignment focuses the processing power on RNs with only a few connected VNs. This is because, as discussed in Section II, such RNs are less complex to process and their soft information contributes more to the detection confidence than these RNs with many connected VNs. Second, we propose the VN deactivation process that, based on the the soft information of the assigned RN's and similar to [1], identifies the VNs that have reached a high decoding confidence (i.e., a large LLR amplitude) and deactivates them. This effectively reduces the number of connections between the VNs and the RNs and consequently reduces the processing requirements of the corresponding nodes. As a result, for given complexity limitations, a larger amount of RNs can be assigned for processing and processed in later iterations. Exploiting the soft-information provided by these additionally processed RNs, more VNs can reach a state of high decoding confidence. This, further reduces the processing requirements and enables the assignment of even more RNs in following iterations. As shown in Section $\mathrm{IV}$, this synergy between the proposed $\mathrm{RN}$ assignment and VN deactivation enables a detection performance similar to the one of full MPA but at a complexity which can be up to two orders of magnitude less. It is worth noting that, neither the $\mathrm{RN}$ assignment nor the $\mathrm{VN}$ deactivation can alone enable consistent and effective multiuser detection for large scale grant free access systems. In particular, a detector that only exploits a VN deactivation approach can suffer from prohibitively high complexity in early iterations, similarly to [4], [6], [3]. On the other hand, a system that employs only RN assignment can meet any given complexity limitations, but this can result in processing only a small fraction of $\mathrm{RNs}$, and therefore in poor detection performance. When, the processing limitations are modeled in terms of maximum Euclidean distance calculations (EDCs) per iteration $N_{E D}$, the scheme works as follows:

RN assignment: $R N$ assignment takes place at the beginning of each decoding iteration, before the traditional RN and VN processing. First, the number of allowable EDCs, denoted as $\mathcal{E}$, is set to $N_{E D}$. Then, the proposed algorithm appoints each RN to a group $G_{v}$ based on the number of its connections to active VNs, denoted as $v$. Next, the total number of EDCs required to process all RNs in the group with the lowest $v$ is calculated as

$$
N_{G_{v}}=\left|G_{v}\right| \cdot 2^{M \cdot v} .
$$

When $N_{G_{v}}$ is smaller than $\mathcal{E}$, all the RNs in the evaluated group are assigned for processing and the algorithm updates the available EDCs to $\mathcal{E}=\mathcal{E}-N_{G_{v}}$. This procedure is repeated for the remaining groups in an increasing order of their $v$ values until either all RNs are assigned or the required EDCs of any group $N_{G_{v}}$ exceeds $\mathcal{E}$. If $N_{G_{v}}>\mathcal{E}$ the algorithm assigns randomly $r$ RNs of the corresponding group, with

$$
r=\left\lfloor\frac{\mathcal{E}}{2^{M \cdot v}}\right\rfloor \text {. }
$$

This, maximizes the number of assigned RNs for the given complexity limitations. We note that this random selection of RNs allows visiting new RNs at every iteration, even if no additional VNs have been deactivated, enabling more VNs to reach a high detection confidence. The pseudocode for the above RN assignment procedure is outlined in Algorithm 1.

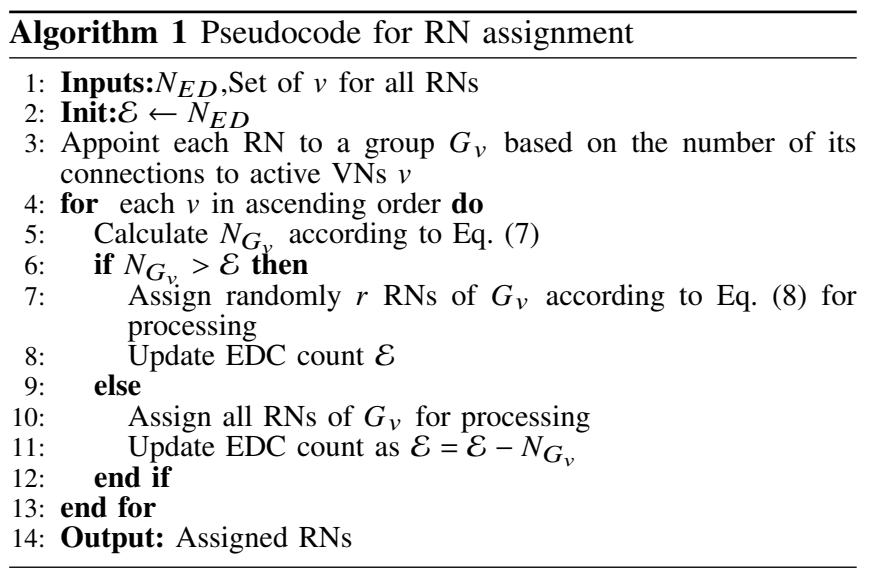

$V N$ deactivation: VN deactivation takes place at the end of each decoding iteration, and after the traditional RN and $\mathrm{VN}$ processing. As discussed, the $\mathrm{VN}$ deactivation uses the already calculated log likelihoods (5) to easily identify reliable codewords. In this context, we assume a codeword is reliable when its probability to be erroneous is below a given threshold 


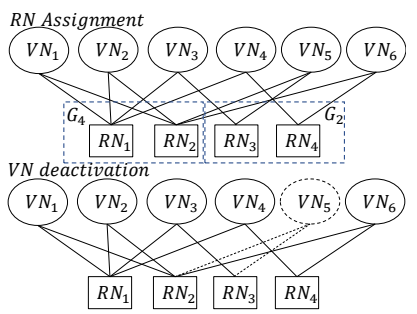

Fig. 2: Proposed MPA decoding graph for unsupervised grand free access

$\delta$, with $\delta \leq 1$ and $\delta$ being a design parameter. In detail, we characterize a codeword $\mathbf{x}_{c} \in C_{q}$ to be highly reliable when

$$
\begin{aligned}
& P\left(\mathbf{x}_{c}=\mathbf{x}^{*}\right)>1-\delta \\
\Leftrightarrow & \frac{P\left(\mathbf{x}_{c}=\mathbf{x}^{*}\right)}{P\left(\mathbf{x}_{c} \neq \mathbf{x}^{*}\right)}>\frac{\delta}{P\left(\mathbf{x}_{c} \neq \mathbf{x}^{*}\right)}>\frac{\delta}{1-\delta},
\end{aligned}
$$

where $\mathbf{x}^{*}$ is the transmitted codeword. Since, $\delta \leq 1$, in (9), the expressions on both sides of the inequality are non-negative. Therefore,

$$
\log \left(\frac{P\left(\mathbf{x}_{c}=\mathbf{x}^{*}\right)}{P\left(\mathbf{x}_{c} \neq \mathbf{x}^{*}\right)}\right)>\log \left(\frac{\delta}{1-\delta}\right) .
$$

Applying the max-log approximation, the left part of (11) can be expressed as

$$
\log \left(\frac{P\left(\mathbf{x}_{c}=\mathbf{x}^{*}\right)}{P\left(\mathbf{x}_{c} \neq \mathbf{x}^{*}\right)}\right) \approx \log \left(\frac{P\left(\mathbf{x}_{c}=\mathbf{x}^{*}\right)}{\max _{\mathbf{x} \in C_{q} \cdot \mathbf{x} \neq \mathbf{x}_{c}} P\left(\mathbf{x}=\mathbf{x}^{*}\right)}\right) .
$$

Then, by employing the relationship of (12), (11) can be expressed in terms of MPA-computed soft messages $I_{q}\left(\mathbf{x}_{c}\right)=$ $\log \left(P\left(\mathbf{x}_{c}=\mathbf{x}^{*}\right)\right.$ as

$$
\left(I_{q}\left(\mathbf{x}_{c}\right)-\max _{\mathbf{x} \in C_{q} \cdot \mathbf{x} \neq \mathbf{x}_{c}} I_{q}(\mathbf{x})\right)>\log \left(\frac{\delta}{1-\delta}\right) .
$$

To avoid repeatedly evaluating the right hand expression in (13), we define the auxiliary variable $\tau=\log ((\delta) /(1-\delta))$. and the proposed algorithm classifies the codeword $\mathbf{x}_{c} \in C_{q}$ as highly reliable when

$$
\left(I_{q}\left(\mathbf{x}_{c}\right)-\max _{\mathbf{x} \in C_{q} \cdot \mathbf{x} \neq \mathbf{x}_{c}} I_{q}(\mathbf{x})\right)>\tau .
$$

After deactivating $V N_{q}$, the algorithm passes the identified highly reliable codeword $\mathbf{x}_{c}$ to all connected RNs, which will use it as reference for performing hard interference cancellation as in [6]. After interference cancellation, the complexity requirements of the corresponding RNs are reduced by a factor of $2^{M}$. The whole process is repeated during the next iteration.

Fig. 2 illustrates an exemplary decoding process with six users $(U=6)$ that concurrently transmit two information bits $(M=2)$ on four subcarriers $(K=4)$. Due to the the unsupervised user access, the subcarrier utilization is asymmetric and $R N_{1}$ and $R N_{2}$ are occupied by four users while $R N_{3}$ and $R N_{4}$ are occupied by two. As an example we set $N_{E D}=350$, and employ the $\mathrm{RN}$ assignment procedure explained in the pseudocode of Algorithm 1. In the first iteration, the RN assignment allocates the RNs to the groups $G_{2}=\left[R N_{3}, R N_{4}\right]$ and $G_{4}=\left[R N_{1}, R N_{2}\right]$ since $R N_{3}$ and $R N_{4}$ are occupied by two users (e.g., $v=2$ ) while $R N_{1}$ and $R N_{2}$ are occupied by four (e.g., $v=4)$ as shown in the example of Fig. 2. Using
(7) we calculate that $N_{G_{2}}=32$, in the ascending order of $v$. Since $N_{G_{2}}<\mathcal{E}$, the nodes $R N_{3}$ and $R N_{4}$ are assigned for processing and $\mathcal{E}$ is updated to $350-32=318$. Then, the algorithm proceeds with calculating $N_{G_{4}}=512$. Since, $N_{G_{4}}>\mathcal{E}$ not all RNs in $N_{G_{4}}$ can be processed. Hence, using (8) we calculate that $r=1$, meaning only one $\mathrm{RN}$ in $N_{G_{4}}$ can be assigned. By assuming that $R N_{2}$ has been randomly selected for processing, first the assigned $\mathrm{RNs}\left(R N_{2}, R N_{3}\right.$ and $R N_{4}$ ) and then all VNs are processed. Assuming that in the given example one codeword $x_{c} \in C_{5}$ reaches an LLR amplitude that fulfills condition (14), $V N_{5}$ will be deactivated after the first iteration. Hence, the complexity requirements of the connected nodes $R N_{2}$ and $R N_{3}$ are reduced (by a factor of $2^{M}=4$ ) to 64 and 4 respectively. Consequently, the total required complexity to process all RNs is $256+64+4+16=340$ EDCs, which is is smaller than $N_{E D}=350$ and, therefore, all RNs can be processed in the following iteration.

\section{Simulation Results}

We evaluate the error rate performance of the proposed SCMA detection algorithm for both coded and uncoded transmissions. The channel coefficients for all users and across all subcarriers are assumed to be i.i.d. complex Gaussian with zero mean and variance of one. For the uncoded scenarios we assume independent channel realizations and independent codebook selection per channel use. For the coded scenarios block fading transmission is assumed, and both the channel realization and codebook are kept constant within one block transmission. Low density parity check (LDPC) codes, which have been recently selected as forward error correction (FEC) for data channels in 3GPP's new radio, with a block length of 624 bits are used for channel coding. An overloading factor of 1.5 is assumed for all simulations. Since the SCMA codebook design for large scale grant-free access with asymmetric resource utilization is an open problem, we adopt a codebook design with the corresponding $g_{C}$ mapping the information bit vector b onto $N$ identical 4-QAM symbols. To compare setups with different numbers of OFDM subcarriers we normalize the maximum allowable number of EDCs per detection iteration $N_{E D}$ to the number of available subcarriers $K$, and we refer to it as $n_{E D}$ (where $n_{E D}=N_{E D} / K$ ). The parameter $\tau$ is set to 4.6 , which equals a likelihood ratio of 100 between the most promising and second most promising codewords of a codebook. Such a large ratio allows for a complexity reduction only when the detector is extremely confident about the detected codeword and, as a result, it preserves system's PER performance. As we have validated via simulations, small deviations of the $\tau$ value around 4.6 do not significantly affect our scheme's complexity/performance trade-off.

Fig. 3 shows the uncoded bit error rate (BER) curves for the proposed approach against the traditional MPA, for various complexity constraints, and for 24 interfering users. When MPA's complexity requirements exceed the given constraints, the received signal cannot be processed and all the transmitted bits are lost, resulting in in impractical error floors. Contrarily, the proposed method consistently reduces the BER when increasing the SNR and when relaxing the complexity constraints. In addition, when increasing the $n_{E D}$ value, the 


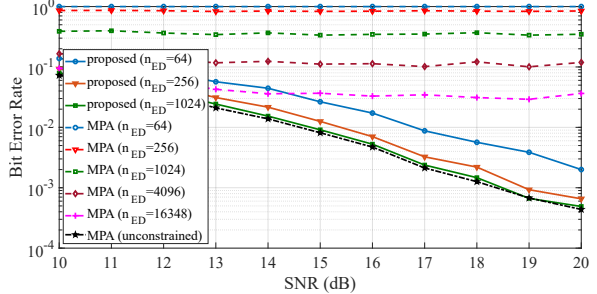

Fig. 3: Uncoded bit error rates of the proposed approach (solid) and traditional MPA (dashed) with various complexity constraints for a grant-free SCMA system with 24 interfering users.

performance of the proposed algorithm asymptotically approaches the performance of an unconstrained MPA decoder. For example, the SNR loss of the proposed approach with $n_{E D}=1024$, is less than $0.25 \mathrm{~dB}$ compared to unconstrained MPA. Fig. 4 compares the complexity, in terms of $n_{E D}$, and the coded Packet-Error-Rate (PER) of the proposed approach with traditional MPA decoder whose complexity is bounded to $n_{E D}=65536$. The proposed approach can provide complexity reductions of more than two orders of magnitude for a negligible PER performance degradation.
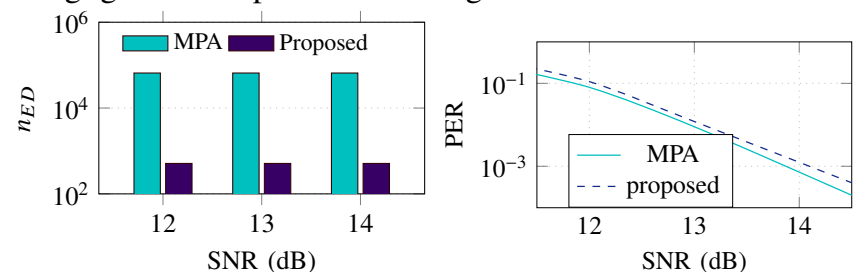

Fig. 4: Complexity and PER performance trade-off for a unsupervised SCMA system with $K=64$ and $U=96$.

Fig. 5 shows the coded PER for the proposed approach and the traditional MPA under various complexity constraints and for several numbers of interfering users, at an SNR of $14 \mathrm{~dB}$, with the proposed method substantially outperforming traditional MPA in all evaluated scenarios. The simulation results also reveal that the PER of MPA increases when the number of users increases and the processing constraints remain constant. When the complexity of MPA is limited to $n_{E D}=1024$, six interfering users can be decoded with an average PER of $10 \%$. However, if the interfering users become 324, the PER increases to $90 \%$. Fig. 5 shows that not only the proposed scheme consistently outperforms the complexity constrained MPA, but also the PER of the proposed approach decreases when the number of subcarriers/users increases. This is because when the number of subcarriers increases, the number of related codebooks increases exponentially but the number of users increases just linearly. As a result, when the number of subcarriers/users increases, it is less likely to have situations where a large number of users share the exact same codebook, which would degrade the performance of the proposed approach. Fig. 5 further shows that the proposed method reaches a PER of 0.1 for 324 users and for an $n_{E D}$ of only 64. While, due to practical limitations, we cannot simulate the PER for extremely high $n_{E D}$ values, we still see that the traditional MPA requires an $n_{E D}$ of 6994 to be able to process $90 \%$ of the possible codebook combinations. This is a necessary (but still not a sufficient) condition for MPA to reach

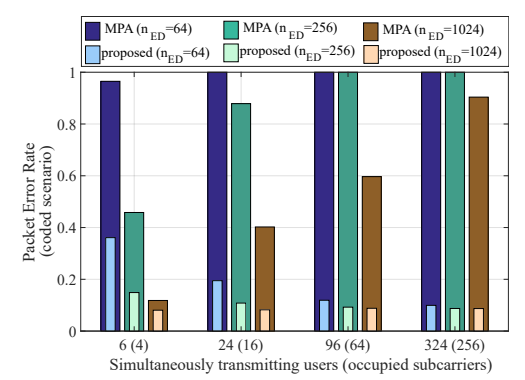

Fig. 5: PER of the proposed approach (thin bars) and traditional MPA (thick bars) for various complexity constraints and numbers of interfering users at $14 \mathrm{~dB}$ SNR

a PER of $10 \%$. Hence, for large numbers of interfering users, the proposed approach can outperform traditional MPA, while still being more than two orders of magnitude less complex.

\section{CONCLUSION}

A simple MPA-based detection approach is proposed that enables multi-user detection of hundreds of mutually interfering information streams in large scale "unsupervised" grantfree access systems. The proposed approach can support hundreds of mutually interfering users with computational requirements that are orders of magnitude smaller than those of traditional MPA-based approaches.

\section{REFERENCES}

[1] H. Nikopour and H. Baligh, "Sparse code multiple access," in Proc. PIMRC. IEEE, 2013, pp. 332-336.

[2] F. R. Kschischang, B. J. Frey, and H.-A. Loeliger, "Factor graphs and the sum-product algorithm," IEEE Trans. Inf. Theory, vol. 47, no. 2, pp. 498-519, 2001.

[3] F. Wei and W. Chen, "Low complexity iterative receiver design for sparse code multiple access," IEEE Trans. Commun., vol. 65, no. 2, pp. 621634, 2017.

[4] S. Zhang, X. Xu, L. Lu, Y. Wu, G. He, and Y. Chen, "Sparse code multiple access: An energy efficient uplink approach for $5 \mathrm{G}$ wireless systems," in Proc. GLOBECOM. IEEE, 2014, pp. 4782-4787.

[5] A. Bayesteh, H. Nikopour, M. Taherzadeh, H. Baligh, and J. Ma, "Low complexity techniques for SCMA detection," in Proc. GLOBECOM. IEEE, 2015, pp. 1-6.

[6] H. Mu, Z. Ma, M. Alhaji, P. Fan, and D. Chen, "A fixed low complexity message pass algorithm detector for up-link SCMA system," IEEE Wireless Commun. Lett., vol. 4, no. 6, pp. 585-588, 2015.

[7] K. Nikitopoulos and G. Ascheid, "Approximate MIMO iterative processing with adjustable complexity requirements," IEEE Trans. Veh. Technol., vol. 61, no. 2, pp. 639-650, 2012.

[8] K. Nikitopoulos, G. Georgis, C. Jayawardena, D. Chatzipanagiotis, and R. Tafazolli, "Massively parallel tree search for high-dimensional sphere decoders," IEEE Trans. Parallel Distrib. Syst, 2018.

[9] C. Jayawardena and K. Nikitopoulos, "G-multisphere: Generalizing massively parallel detection for non-orthogonal signal transmissions," IEEE Trans. on Commun., vol. 68, no. 2, pp. 1227-1239, 2020.

[10] K. Au, L. Zhang, H. Nikopour, E. Yi, A. Bayesteh, U. Vilaipornsawai, J. Ma, and P. Zhu, "Uplink contention based SCMA for 5G radio access," in Proc. GLOBECOM. IEEE, 2014, pp. 900-905.

[11] A. Bayesteh, E. Yi, H. Nikopour, and H. Baligh, "Blind detection of SCMA for uplink grant-free multiple-access," in Proc. Wireless Commun. Syst. (ISWCS). IEEE, 2014, pp. 853-857.

[12] J. Zhang, L. Lu, Y. Sun, Y. Chen, J. Liang, J. Liu, H. Yang, S. Xing, Y. Wu, J. Ma et al., "PoC of SCMA-based Uplink grant-free transmission in UCNC for 5G," IEEE J. Sel. Areas in Commun., 2017.

[13] R. Hoshyar, F. P. Wathan, and R. Tafazolli, "Novel low-density signature for synchronous CDMA systems over AWGN channel," IEEE Trans. Signal Process., vol. 56, no. 4, pp. 1616-1626, 2008. 